\title{
Journal of Diabetic Complications \&

\section{The Endothelial Progenitor Cell Dysfunction in Type 2 Diabetes Mellitus: The Link with Heart Failure Developing}

\section{Alexander $\mathbf{B}^{*}$}

Consultant of Therapeutic Unit, Internal Medicine Department, State Medical University for Zaporozhye, Mayakovsky, Ukraine

*Corresponding author: Alexander B, Consultant of Therapeutic Unit, Internal Medicine Department, State Medical University for Zaporozhye, Mayakovsky, Ukraine, Tel: +380612894585; E-mail: aeberezin@gmail.com

Received date: May 17, 2018; Accepted date: May 18, 2018; Published date: May 23, 2018

Copyright: () 2018 Alexander B. This is an open-access article distributed under the terms of the Creative Commons Attribution License, which permits unrestricted use, distribution, and reproduction in any medium, provided the original author and source are credited.

\begin{abstract}
The circulating number of Endothelial Progenitor Cells (EPCs) decrease in patients with established heart failure (HF) depending the severity of the disease and may reflect poor prognosis. Developing type 2 diabetes mellitus (T2DM) associates with decreased number and weak function of EPCs that corresponds to impaired vascular repair and endothelial dysfunction. It is well known that T2DM is a risk factor of HF, but the role of EPC dysfunction in T2DM-induced HF is not fully investigated. The short communication is depicted a role of decreased circulating number and lowered function of EPCs in T2DM patients as a predictive biological marker of HF manifestation. It has been suggested that the potential use of EPCs as a novel personified indicator of cardiac dysfunction in diabetics is challenging and requires being under scrutinizes in future.
\end{abstract}

Keywords: Diabetes mellitus; Endothelial progenitor cells; Heart failure; Biomarkers

\section{Introduction}

Diabetes mellitus (DM) and pre-diabetes are established risk factors of premature cardiovascular $(\mathrm{CV})$ disease and events including heart failure (HF) [1,2]. There are numerous innate molecular mechanisms that are involved in the pathogenesis of cardiac dysfunction in type 2 DM (T2DM), majority all of them are typically attributed to poor hyperglycemia control, metabolic memory phenomenon, dyslipidemia, oxidative stress, low-grade systemic and micro vascular inflammation, accelerating atherosclerosis, vasculopathy and ischemia [3]. Therefore, complexed contribution of several co-morbidities, such as hypertension, atherogenic hyperlipidemia, abdominal obesity, renal insufficiency, can be seized upon the triggers of different $\mathrm{HF}$ phenotypes in T2DM [4]. Although altered insulin-related signaling pathways and imbalance in ERK1/2 and PKCa activity in T2DM are considered a leading causes that modifying cardiac contractile and structure proteins (titin, SERCA, troponins) through mediate cardiomyocyte tension/ injury and biochemical stress and thereby contributing to diastolic/systolic dysfunction $[5,6]$ impaired tissue reparation remains large unknown factor corresponding to modification of genome circulating progenitors and residence cells [7].

Endothelial progenitor cells (EPCs) are defined as CD45(-) adherent cells derived from peripheral blood- or bone marrow-derived mononuclear cells that co-expressing endothelial cell antigens (CD34, CD133, CD309) and demonstrating isolectin-binding capacity and ability to appear in fibronectin coated dish [8]. Some populations of EPCs may lose CD133 antigen within differentiation period and may express on their surface other antigens, i.e. CD31, CD144, endothelial NO synthase and von Willebrand factor [9]. All EPCs were divided into early outgrowth EPCs or late outgrowth EPCs [10]. The early EPCs originated from the hematopoietic lineage with a limited proliferative capability and emerged after 4 to 7 days of culture. However, they contribute to neovascularization via secretion of proangiogenic cytokines in vivo. Late EPCs appear in culture in 1 to 3 weeks and obligatory express CD34 with other endothelial markers, such as CD309 and CD146. Depending on presentation on the cell surface CD34 antigen all late outgrowth EPCs are divided into two populations. The population of EPCs with immune phenotypes CD34(+)CD31(+), CD34(+)CD31(+)CD146(+), CD34(+)CD31(+), $\mathrm{CD} 105(+)$, and $\mathrm{CD} 34(+) \mathrm{CD} 31(+) \mathrm{CD} 309(+)$ demonstrated higher proliferative potency to CD34(-) EPCs co-expressing CD31, CD309, CD105 and CD146 antigens. Therefore, CD34(+) EPCs had reproduced tubes and colony shaping in the single-cell colonyformation investigation as well as they responded to angiogenic growth factors [11].

The decrease number and reduced function of EPCs are defined as EPC dysfunction. Figure is reported the basic innate molecular mechanisms leading to EPC dysfunction. Interestingly, an effect of early T2DM and late T2DM on pattern and functionality of EPCs sufficiently varies [12]. Indeed, metabolic abnormalities that are resulting in developing of T2DM can modify function and decrease numbers of EPCs by various means acting as epigenetic regulator, while at early stage of T2DM in young patients the number of EPCs may even temporary increase [13]. Both experimental and clinical studies have yielded that hyperglycemia alone related to T2DM was primary trigger that suppressed the Akt/eNOS pathway and thereby alters the proliferative ability of EPCs [14]. Therefore, hyperglycemia, oxidative stress metabolites and inflammatory cytokines including adipocytokines decreased the production of granulocyte colonystimulating factor, stem cell factor, and vascular endothelial growth factor (VEGF), which reduced the local level of chemo attractant and impaired mobilization of pluripotent cells from bone marrow via the cleavage of stromal-derived factor 1 (SDF-1 $\alpha$ ) through the release of protease CD26 and altered expression of predominantly microRNA 126 and microRNA 130a [14,15]. Additionally, there is evidence that traditional CV risk factors, such as uric acid, pro-inflammatory adipocytokines, oxidized lipids, can block SDF-1 $\alpha$ and or its receptor CXCR4, suppress extracellular signal-regulated kinase, VEGF, and the phosphoinositide 3-kinase/ Akt/eNOS signal pathway in EPCs and 
thereby prevent the recruitment of EPCs from bone marrow to injured sites [13].

Thus, combined influences of hyperglycemia in cooperation with oxidative stress components (advanced glycation end products, reactive oxidative species, and thrombospondin 2), oxidized lipids, inflammatory cytokines (interleukin-2, interleukin-8, adiponectin, vistafin) damage Akt/eNOS/nuclear factor- $\mathrm{kB}$ signal transduction in target cells. All these lead to mitochondrial dysfunction, altered nucleotide metabolism and impaired chromatin (telomere shortening, oxidative DNA damage) in the EPCs thereby worsening their ability to differentiation, proliferation, moving, and survival [16]. As a result, modified EPCs cannot (trans)-differentiate into mature endothelial cells and restore integrity of endothelium and vascular function [17]. On the other hand, modified EPCs frequently synthase and realize micro vesicles with micro RNAs, modified DNAs and active molecules that directly injury structure and function of target cells, such as immature hemoangioblasts and tissue resident cells [18]. Therefore, losing ability of modified EPCs to secrete angiogenic growth factors lead to impaired cell-to-cell cooperation and mediate altered vascular reparation particularly limiting trans-differentiation of adventitial cells/immature endothelial progenitors into smooth muscle cells and EPCs into mature endothelial cells $[19,20]$. In fact, neovascularization and angiogenesis supporting by EPCs are sufficiently decrease in T2DM and they can be a pivotal factors contributing to $\mathrm{CV}$ complications and HF [21]. Indeed, the number of circulating EPCs inversely associates with the number of CV risk factors [22] and it is reduced in CV disease including HF and T2DM $[23,24]$. There is evidence that not just decreased number of EPCs, but weak function of circulating EPCs may be an additional risk factor with independent ability to predict CV mortality rate, a risk of urgent hospital admission due to HF and newly diagnosed HF in patients with pre-diabetes and established T2DM [25]. Interesting, the lowered function of EPCs appeared prior to dramatically decreased number of EPCs in young patients with T2DM without established CV disease and HF, while in T2DM-induced HF there was significantly lowered number and weak function of circulating EPCs [25]. Whether this fact would be useful to differentiate diabetics at risk of HF manifestation is not fully clear and requires to be investigated in large clinical studies. In fact, the measurement of circulating number and assay function of EPCs may serve as a surrogate biological marker for T2DM patients at higher risk HF and HF-related complications [26]. Multiple molecular mechanisms include several changes in Akt/eNOS/nuclear factor- $\kappa B$ signal transduction, altered expression of inflammatory-induced genes (CRP, interleukin 2 and 8), increased oxidative stress (increased ROS, AGEs/RAGEs), modification of chromatin of precursors, impaired mitochondrial function of the EPCs that mediate impairment in their homing, differentiation, mobilization, trafficking and survival in the circulation (Figure 1).

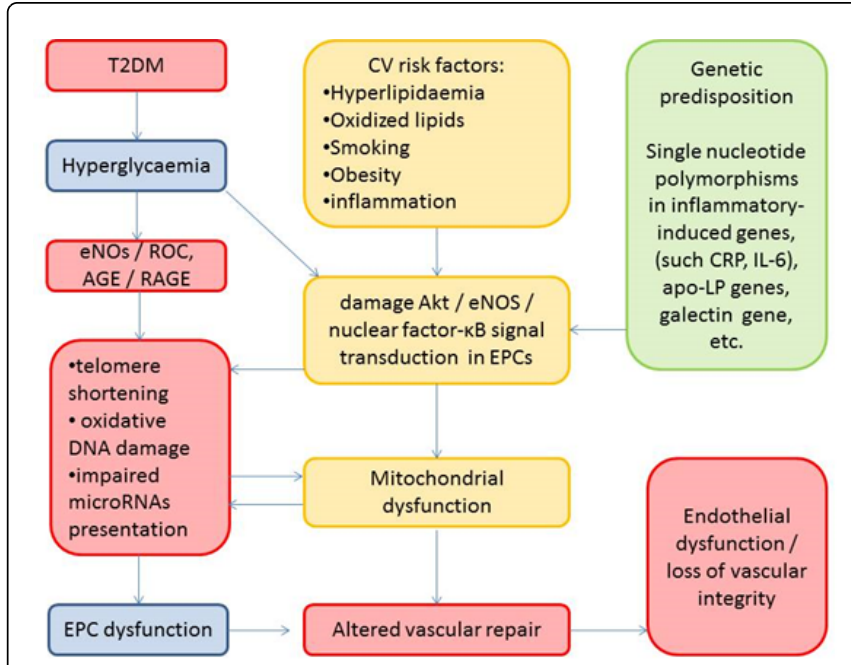

ENOS: Endothelial nitric oxide synthase; A GEs: Advanced glycation end products;

RAGEs: Receptor for advanced glycation end products; EPCs: endothelial progenitor cells; ROS: Reactive oxidative species; LP: Lipoproteins; CRP: C-reactive protein; IL: Interleukins; T2DM: Type 2 diabetes mellitus.

Figure 1: The potential molecular mechanisms leading to EPC dysfunction in diabetics.

\section{Conclusion}

There are multiple factors that affect number and function of circulating EPCs in T2DM and T2DM-induced HF. However, the potential use of EPCs as a novel personified biological marker of cardiac dysfunction in diabetics is challenging and requires being under scrutinizes in future.

\section{References}

1. Meagher P, Adam M, Civitarese R, Bugyei TA (2018) Connelly KA. Heart Failure With Preserved Ejection Fraction in Diabetes: Mechanisms and Management. Can J Cardiol 34: 632-643.

2. Kotsis V, Jordan J, Micic D, Finer N, Leitner DR, et al (2018) Obesity and cardiovascular risk: a call for action from the European Society of Hypertension Working Group of Obesity, Diabetes and the High-risk Patient and European Association for the Study of Obesity: part A: mechanisms of obesity induced hypertension, diabetes and dyslipidemia and practice guidelines for treatment. J Hypertens 1: 1-4.

3. Pedro BJ, Chillarón JJ, Benaiges D, Flores-Le Roux JA (2016) Cardiovascular prevention in diabetes mellitus: A multifactorial challenge. Clin Investig Arterioscler 28:1 54-63.

4. Arrieta F, Iglesias P, Pedro-Botet J, Becerra A, Ortega E, et al (2018) Grupo de Riesgo Cardiovascular de la Sociedad Española de Diabetes (SED). Diabetes mellitus and cardiovascular risk: Update of the recommendations of the Diabetes and Cardiovascular Disease working group of the Spanish Diabetes Society. Clin Investig Arterioscler 22: 115-121.

5. Hopf AE, Andresen C, Kötter S, Isic M, Ulrich K, et al (2018) DiabetesInduced Cardiomyocyte Passive Stiffening Is Caused by Impaired InsulinDependent Titin Modification and Can Be Modulated by Neuregulin-1. Circ Res 3: 112-115.

6. Berezin AE (2017) Cardiac biomarkers in diabetes mellitus: New dawn for risk stratification?. Diabetes Metab Syndr 11: 201-208. 
Citation: $\quad$ Alexander B (2018) The Endothelial Progenitor Cell Dysfunction in Type 2 Diabetes Mellitus: The Link with Heart Failure Developing. J Diabetic Complications Med 3: 121. doi:10.4172/2475-3211.1000121

Page 3 of 3

7. Berezin AE (2017) Endothelial progenitor cells dysfunction and impaired tissue reparation: The missed link in diabetes mellitus development. Diabetes Metab Syndr 11:215-220.

8. Haberzettl P, Conklin DJ, Toole TE (2018) Endothelial Progenitor Cells: Properties, Function, and Response to Toxicological Stimuli. Comprehensive Toxicology 12: 130-182.

9. Boldicke T, Tesar M, Griesel C, Rohde M, Gröne HJ, et al (2001) AntiVEGFR-2 scFvs for cell isolation. Single-chain antibodies recognizing the human vascular endothelial growth factor receptor-2 (VEGFR-2/flk-1) on the surface of primary endothelial cells and preselected CD34+ cells from cord blood. Stem Cells 19: 24-36.

10. $\mathrm{Xu} \mathrm{QB}$, Sheng $\mathrm{Li}$, Xue Bao (2005) Endothelial progenitor cells in angiogenesis. Therapeutic Angiogenesis 57: 1-6.

11. Ferreras C, Cole CL, Urban K, Jayson GC, Avizienyte E (2015) Segregation of late outgrowth endothelial cells into functional endothelial CD34- and progenitor-like CD34+ cell populations. Angiogenesis 18: 47-68.

12. Berezin A (2016) Metabolic memory phenomenon in diabetes mellitus: Achieving and perspectives. Diabetes Metab Syndr 10: 176-83.

13. Berezin AE (2016) Epigenetics in heart failure phenotypes. BBA Clinical 6: 31-37.

14. Fadini GP, Boscaro E, de Kreutzenberg S, Agostini C, Seeger F, et al (2010) Time course and mechanisms of circulating progenitor cell reduction in the natural history of type 2 diabetes. Diabetes Care 33 : 1097-1102.

15. Churdchomjan W, Kheolamai P, Manochantr S, Tapanadechopone P, Tantrawatpan C, et al (2010) Comparison of endothelial progenitor cell function in type 2 diabetes with good and poor glycemic control. BMC Endocr Disord 10: 1-5.

16. Tatekoshi Y, Tanno M, Kouzu H, Abe K, Miki T, et al (2018) Translational regulation by miR-301b upregulates AMP deaminase in diabetic hearts. J Mol Cell Cardiol 119: 138-146.
17. Berezin AE (2016) Metabolomics in Heart Failure Patients: Hype and Hope. J Biomarkers 2: 21-23.

18. Berezin AE (2016) Impaired immune phenotype of endothelial cellderived microparticles: the missed link between diabetes-related states and cardiovascular complications. J Data Mining Genomics \& Proteomics 7: 195-197.

19. Rehman J, Li J, Orschell CM, March KL (2003) Peripheral blood endothelial progenitor cells are derived from monocyte/macrophages and secrete angiogenic growth factors. Circulation 107: 1164-169.

20. Berezin AE, Kremzer AA, Berezina TA, Martovitskaya YV (2016) The pattern of circulating microparticles in patients with diabetes mellitus with asymptomatic atherosclerosis. Acta Clinica Belgica. International Journal of Clinical and Laboratory Medicine 71: 38-45.

21. Yiu KH, Tse HF (2014) Specific role of impaired glucose metabolism and diabetes mellitus in endothelial progenitor cell characteristics and function. Arterioscler Thromb Vasc Biol 34: 1136-143.

22. Vasa M, Fichtlscherer S, Aicher A, Adler K, Urbich C, et al (2001) Number and migratory activity of circulating endothelial progenitor cells inversely correlate with risk factors for coronary artery disease. Circ Res 89: 1-7.

23. Berezin AE (2016) Prognostication in Different Heart Failure Phenotypes: The Role of Circulating Biomarkers. J Circ Biomark 6: 5-6.

24. Fadini GP, Sartore S, Baesso I, Lenzi M, Agostini C, et al (2006) Endothelial progenitor cells and the diabetic paradox. Diabetes Care 29: 714-16.

25. Berezin AE, Kremzer AA (2014) Relationship between circulating endothelial progenitor cells and insulin resistance in non-diabetic patients with ischemic chronic heart failure. Diabetes Metab Syndr 8: 138-144.

26. Berezin AE, Kremzer AA, Martovitskaya YuV, Samura TA, Berezina TA (2014) The predictive role of circulating microparticles in patients with chronic heart failure. BBA Clinical 3: 18-24. 